International Mathematical Forum, 2, 2007, no. 31, 1499 - 1506

\title{
Common Fixed Point Theorems for Multi-valued Contractions
}

\author{
S. Sedghi ${ }^{1}$ \\ Department of Mathematics \\ Islamic Azad University-Ghaemshahr Branch \\ Ghaemshahr P.O. Box 163, Iran \\ sedghi_gh@yahoo.com \\ N. Shobe \\ Department of Mathematics \\ Islamic Azad University-Babol Branch, Iran \\ nabi_shobe@yahoo.com
}

\begin{abstract}
In this paper, a common fixed point theorem for weakly compatible maps in fuzzy metric spaces is proved.

Mathematics Subject Classification: 54E40; 54E35; 54H25

Keywords: Common fixed points; weakly compatible mappings; Complete fuzzy metric space

\section{Introduction And Preliminaries}

The concept of fuzzy sets was introduced initially by Zadeh [17] in 1965. Since then, to use this concept in topology and analysis many authors have expansively developed the theory of fuzzy sets and application. George and Veeramani [7] and Kramosil and Michalek [11] have introduced the concept of fuzzy topological spaces induced by fuzzy metric which have very important applications in quantum particle physics particularly in connections with both string and $E$-infinity theory which were given and studied by El Naschie [1, 2, $3,4,16]$. Many authors $[8,13,14]$ have proved fixed point theorem in fuzzy (probabilistic) metric spaces.
\end{abstract}

Definition 1.1. A binary operation $*:[0,1] \times[0,1] \longrightarrow[0,1]$ is a continuous t-norm if it satisfies the following conditions

1. $*$ is associative and commutative,

\footnotetext{
${ }^{1}$ Corresponding author: sedghi_gh@yahoo.com
} 
2. $*$ is continuous,

3. $a * 1=a$ for all $a \in[0,1]$,

4. $a * b \leq c * d$ whenever $a \leq c$ and $b \leq d$, for each $a, b, c, d \in[0,1]$.

Two typical examples of continuous t-norm are $a * b=a b$ and $a * b=$ $\min (a, b)$.

Definition 1.2. A 3-tuple $(X, M, *)$ is called a fuzzy metric space if $X$ is an arbitrary (non-empty) set, $*$ is a continuous t-norm, and $M$ is a fuzzy set on $X^{2} \times(0, \infty)$, satisfying the following conditions for each $x, y, z \in X$ and $t, s>0$,

1. $M(x, y, t)>0$,

2. $M(x, y, t)=1$ if and only if $x=y$,

3. $M(x, y, t)=M(y, x, t)$,

4. $M(x, y, t) * M(y, z, s) \leq M(x, z, t+s)$,

5. $M(x, y,):.(0, \infty) \longrightarrow[0,1]$ is continuous.

Let $(X, M, *)$ be a fuzzy metric space. For $t>0$, the open ball $B(x, r, t)$ with center $x \in X$ and radius $0<r<1$ is defined by

$$
B(x, r, t)=\{y \in X: M(x, y, t)>1-r\} .
$$

Let $(X, M, *)$ be a fuzzy metric space. Let $\tau$ be the set of all $A \subset X$ with $x \in A$ if and only if there exist $t>0$ and $0<r<1$ such that $B(x, r, t) \subset A$. Then $\tau$ is a topology on $X$ (induced by the fuzzy metric $M$ ). This topology is Hausdorff and first countable. A sequence $\left\{x_{n}\right\}$ in $X$ converges to $x$ if and only if $M\left(x_{n}, x, t\right) \rightarrow 1$ as $n \rightarrow \infty$, for each $t>0$. It is called a Cauchy sequence if for each $0<\varepsilon<1$ and $t>0$, there exits $n_{0} \in \mathbb{N}$ such that $M\left(x_{n}, x_{m}, t\right)>1-\varepsilon$ for each $n, m \geq n_{0}$. The fuzzy metric space $(X, M, *)$ is said to be complete if every Cauchy sequence is convergent. A subset $A$ of $X$ is said to be F-bounded if there exists $t>0$ and $0<r<1$ such that $M(x, y, t)>1-r$ for all $x, y \in A$.

Lemma 1.3. [7] Let $(X, M, *)$ be a fuzzy metric space. Then $M(x, y, t)$ is non-decreasing with respect to $t$, for all $x, y$ in $X$.

Example 1.4. Let $X=\mathbb{R}$. Denote $a * b=a . b$ for all $a, b \in[0,1]$. For each $t \in] 0, \infty[$, define

for all $x, y \in X$.

$$
M(x, y, t)=\frac{t}{t+|x-y|}
$$

Definition 1.5. Let $(X, M, *)$ be a fuzzy metric space. $M$ is said to be continuous on $X^{2} \times(0, \infty)$ if

$$
\lim _{n \rightarrow \infty} M\left(x_{n}, y_{n}, t_{n}\right)=M(x, y, t) .
$$

Whenever a sequence $\left\{\left(x_{n}, y_{n}, t_{n}\right)\right\}$ in $X^{2} \times(0, \infty)$ converges to a point $(x, y, t) \in$ $X^{2} \times(0, \infty)$ i.e.

$$
\lim _{n \rightarrow \infty} M\left(x_{n}, x, t\right)=\lim _{n \rightarrow \infty} M\left(y_{n}, y, t\right)=1 \text { and } \lim _{n \rightarrow \infty} M\left(x, y, t_{n}\right)=M(x, y, t)
$$


Lemma 1.6. Let $(X, M, *)$ be a fuzzy metric space. Then $M$ is continuous function on $X^{2} \times(0, \infty)$.

Proof. See Proposition 1 of [12].

Lemma 1.7. Let $(X, M, *)$ be a fuzzy metric space. If we define $E_{\lambda, M}: X^{2} \rightarrow$ $\mathbb{R}^{+} \cup\{0\}$ by

$$
E_{\lambda, M}(x, y)=\inf \{t>0: M(x, y, t)>1-\lambda\}
$$

for each $\mu \in(0,1)$ there exists $\lambda \in(0,1)$ such that

$$
E_{\mu, M}\left(x_{1}, x_{n}\right) \leq E_{\lambda, M}\left(x_{1}, x_{2}\right)+E_{\lambda, M}\left(x_{2}, x_{3}\right)+\cdots+E_{\lambda, M}\left(x_{n-1}, x_{n}\right)
$$

for any $x_{1}, x_{2}, \ldots, x_{n} \in X$

(ii) The sequence $\left\{x_{n}\right\}_{n \in \mathbb{N}}$ is convergent in fuzzy metric space $(X, M, *)$ if and only if $E_{\lambda, M}\left(x_{n}, x\right) \rightarrow 0$. Also the sequence $\left\{x_{n}\right\}_{n \in \mathbb{N}}$ is Cauchy sequence if and only if it is Cauchy with $E_{\lambda, M}$.

Proof. (i). For every $\mu \in(0,1)$, we can find a $\lambda \in(0,1)$ such that

$$
\overbrace{(1-\lambda) *(1-\lambda) * \cdots *(1-\lambda)}^{n} \geq 1-\mu
$$

by triangular inequality we have

$$
\begin{aligned}
& \left.M\left(x_{1}, x_{n}, E_{\lambda, M}\left(x_{1}, x_{2}\right)+E_{\lambda, M}\left(x_{2}, x_{3}\right)+\cdots+E_{\lambda, M}\left(x_{n-1}, x_{n}\right)\right)+n \delta\right) \\
& \geq M\left(x_{1}, x_{2}, E_{\lambda, M}\left(x_{1}, x_{2}\right)+\delta\right) * \cdots * M\left(x_{n-1}, x_{n}, E_{\lambda, M}\left(x_{n-1}, x_{n}\right)+\delta\right) \\
& \geq \overbrace{(1-\lambda) *(1-\lambda) * \cdots *(1-\lambda)}^{n} \geq 1-\mu
\end{aligned}
$$

for very $\delta>0$, which implies that

$$
E_{\mu, M}\left(x_{1}, x_{n}\right) \leq E_{\lambda, M}\left(x_{1}, x_{2}\right)+E_{\lambda, M}\left(x_{2}, x_{3}\right)+\cdots+E_{\lambda, M}\left(x_{n-1}, x_{n}\right)+n \delta .
$$

Since $\delta>0$ is arbitrary, we have

$$
E_{\mu, M}\left(x_{1}, x_{n}\right) \leq E_{\lambda, M}\left(x_{1}, x_{2}\right)+E_{\lambda, M}\left(x_{2}, x_{3}\right)+\cdots+E_{\lambda, M}\left(x_{n-1}, x_{n}\right)
$$

(ii). Note that since $\mathrm{M}$ is continuous in its third place and

$$
E_{\lambda, M}(x, y)=\inf \{t>0: M(x, y, t)>1-\lambda\} .
$$

Hence, we have

$$
M\left(x_{n}, x, \eta\right)>1-\lambda \Longleftrightarrow E_{\lambda, M}\left(x_{n}, x\right)<\eta
$$

for every $\eta>0$.

Lemma 1.8. Let $\left(X, M{ }^{*}\right)$ be a fuzzy metric space. If

$$
M\left(x_{n}, x_{n+1}, t\right) \geq M\left(x_{0}, x_{1}, k^{n} t\right)
$$

for some $k>1$ and for every $n \in \mathbb{N}$. Then sequence $\left\{x_{n}\right\}$ is a Cauchy sequence. 
Proof. For every $\lambda \in(0,1)$ and $x_{n}, x_{n+1} \in X$, we have

$$
\begin{aligned}
E_{\lambda, M}\left(x_{n+1}, x_{n}\right) & =\inf \left\{t>0: M\left(x_{n+1}, x_{n}, t\right)>1-\lambda\right\} \\
& \leq \inf \left\{t>0: M\left(x_{0}, x_{1}, k^{n} t\right)>1-\lambda\right\} \\
& =\inf \left\{\frac{t}{k^{n}}: M\left(x_{0}, x_{1}, t\right)>1-\lambda\right\} \\
& =\frac{1}{k^{n}} \inf \left\{t>0: M\left(x_{0}, x_{1}, t\right)>1-\lambda\right\} \\
& =\frac{1}{k^{n}} E_{\lambda, M}\left(x_{0}, x_{1}\right) .
\end{aligned}
$$

By Lemma 1.7, for every $\mu \in(0,1)$ there exists $\lambda \in(0,1)$ such that

$$
\begin{aligned}
E_{\mu, M}\left(x_{n}, x_{m}\right) & \leq E_{\lambda, M}\left(x_{n}, x_{n+1}\right)+E_{\lambda, M}\left(x_{n+1}, x_{n+2}\right)+\cdots+E_{\lambda, M}\left(x_{m-1}, x_{m}\right) \\
& \leq \frac{1}{k^{n}} E_{\lambda, M}\left(x_{0}, x_{1}\right)+\frac{1}{k^{n+1}} E_{\lambda, M}\left(x_{0}, x_{1}\right)+\cdots+\frac{1}{k^{m-1}} E_{\lambda, M}\left(x_{0}, x_{1}\right) \\
& =E_{\lambda, M}\left(x_{0}, x_{1}\right) \sum_{j=n}^{m-1} \frac{1}{k^{j}} \longrightarrow 0 .
\end{aligned}
$$

Hence sequence $\left\{x_{n}\right\}$ is Cauchy sequence.

In 1998, Jungck and Rhoades [10] introduced the following concept of weak compatibility.

Definition 1.9. Let $A$ and $S$ be mappings from a fuzzy metric space $(X, M, *)$ into itself. Then the mappings are said to be weak compatible if they commute at their coincidence point, that is, $A x=S x$ implies that $A S x=S A x$.

Example 1.10. Let $(X, M, *)$ be a fuzzy metric space, in which $\mathrm{X}=[0,2], a *$ $b=\min \{a, b\}$ for all $a, b \in[0,1]$ and $M(x, y, t)=\frac{t}{t+d(x, y)}$ for all $t>0$. Define self-maps $A$ and $S$ on $X$ as follows:

$$
A x=\left\{\begin{array}{ll}
2 & \text { if } 0 \leq x \leq 1, \\
\frac{x}{2} & \text { if } 1<x \leq 2,
\end{array} \quad S x=\left\{\begin{array}{cc}
2 & \text { if } x=1 \\
\frac{x+3}{5} & \text { otherwise }
\end{array}\right.\right.
$$

Then we have $S 1=A 1=2$ and $S 2=A 2=1$. Also $S A 1=A S 1=1$ and $S A 2=A S 2=2$. Thus $(A, S)$ is weak compatible.

Recently, Jungck and Rhoades $[9,10]$ defined the concepts of $\delta$-compatible which extend the concept of compatible mappings in the single-valued setting to set-valued mappings. The following definition is given by Jungck and Rhoades [10].

Definition 1.11. The mappings $I: X \longrightarrow X$ and $F: X \longrightarrow B(X)$ are weakly compatible if they commute at coincidence points, i.e., for each point $u$ in $X$ such that $F u=\{I u\}$, we have $F I u=I F u$. (Note that the equation $F u=\{I u\}$ implies that $F u$ is a singleton). 
Throughout this paper, $B(X)$ is the set of all nonempty bounded subsets of $X$. For every $t>0$ let $\delta(A, B, t)$ be the function defined by

$$
\delta(A, B, t)=\inf \{M(a, b, t) ; a \in A, b \in B\} .
$$

If $A$ consists of a single point $a$, we write $\delta(A, B, t)=\delta(a, B, t)$. If $B$ also consists of a single point $b$, we write $\delta(A, B, t)=M(a, b, t)$.

It follows immediately from the definition that

$$
\begin{aligned}
\delta(A, B, t) & =\delta(B, A, t) \geq 0, \\
\delta(A, B, t+s) & \geq \delta(A, C, t) * \delta(C, B, t), \\
\delta(A, B, t) & =1 \Longleftrightarrow A=B=\{a\},
\end{aligned}
$$

for all $A, B, C$ in $B(X)$.

Lemma 1.12. Let $(X, M, *)$ be a fuzzy metric space. Then $\delta(A, B, t) \leq \delta(A, B, k t)$, for all $A, B$ in $B(X)$ and some $k>1$.

Proof. If $\delta(A, B, t)>\delta(A, B, k t)$ for some $A, B$ in $B(X)$, then for $a \in A$ and $b \in B$ we set $\delta(A, B, k t)=M(a, b, k t)$ for some $k>1$. On the other hand we have

$$
M(a, b, t) \geq \delta(A, B, t)>\delta(A, B, k t)=M(a, b, k t),
$$

which is contradiction.

Definition 1.13. [5]. A sequence $\left\{A_{n}\right\}$ of subsets of $X$ is said to be convergent to a subset $A$ of $X$ if

(i) given $a \in A$, there is a sequence $\left\{a_{n}\right\}$ in $X$ such that $a_{n} \in A_{n}$ for $n=1,2, \cdots$, and $\left\{a_{n}\right\}$ converges to $a$.

(ii) given $\epsilon>0$, there exists a positive integer $N$ such that $A_{n} \subseteq A_{\epsilon}$ for $n>N$ where $A_{\epsilon}$ is the union of all open spheres with centers in $A$ and radius $\epsilon$.

Lemma 1.14. $[6,15]$. Let $\left\{A_{n}\right\}$ be a sequence in $B(X)$ and $y$ a point in $X$ such that $\delta\left(A_{n}, y, t\right) \longrightarrow 1$. Then the sequence $\left\{A_{n}\right\}$ converges to the set $\{y\}$ in $B(X)$.

\section{THE MAIN RESULTS}

We begin by recalling some basic concepts of the main theory of this paper.

Theorem 2.1. Let $F, G$ be mappings of a complete fuzzy metric space $(X, M, *)$ into $B(X)$ and $I, J$ be mappings of $X$ into itself satisfying:

(i) $F(x) \subseteq J(X), G(x) \subseteq I(X)$ for every $x \in X$,

(ii) The pair $(F, I)$ and $(G, J)$ are weakly compatible,

(iii) let $\phi:[0,1]^{3} \longrightarrow[0,1]$, is a continuous function and increasing in any co-ordinate and $\phi(t, t, t)>t$ for every $t \in[0,1)$. 
(iv) $\delta(F x, G y, t) \geq \phi(M(I x, J y, k t), \delta(I x, F x, k t), \delta(J y, G y, k t))$

for every $x, y$ in $X$ and some $k>1$. Suppose that one of $J(X)$ or $S(X)$ is a closed subset of $X$, then there exists a unique $z \in X$ such that $\{z\}=\{I z\}=$ $\{J z\}=F z=G z$.

Proof. Let $x_{0}$ be an arbitrary point in $X$. By $(i)$, we choose a point $x_{1}$ in $X$ such that $J x_{1} \in F x_{0}=Z_{0}$. For this point $x_{1}$ there exists a point $x_{2}$ in $X$ such that $I x_{2} \in G x_{1}=Z_{1}$, and so on. Continuing in this manner we can define a sequence $\left\{x_{n}\right\}$ as follows

$$
J x_{2 n+1} \in F x_{2 n}=Z_{2 n}, I X_{2 n+2} \in G x_{2 n+1}=Z_{2 n+1},
$$

for $n=0,1,2, \cdots$. For simplicity, we put $V_{n}(t)=\delta\left(Z_{n}, Z_{n+1}, t\right)$, for $n=$ $0,1,2, \cdots$. We prove that sequence $\left\{V_{n}(t)\right\}$ is an increasing and convergent to 1. Since

$$
\begin{aligned}
V_{2 n}(t) & =\delta\left(Z_{2 n}, Z_{2 n+1}, t\right)=\delta\left(F x_{2 n}, G x_{2 n+1}, t\right) \\
& \geq \phi\left(M\left(I x_{2 n}, J x_{2 n+1}, k t\right), \delta\left(I x_{2 n}, F x_{2 n}, k t\right), \delta\left(J x_{2 n+1}, G x_{2 n+1}, k t\right)\right) \\
& \geq \phi\left(\delta\left(G x_{2 n-1}, F x_{2 n}, k t\right), \delta\left(G x_{2 n-1}, F x_{2 n}, k t\right), \delta\left(F x_{2 n}, G x_{2 n+1}, k t\right)\right) \\
& =\phi\left(\delta\left(Z_{2 n-1}, Z_{2 n}, k t\right), \delta\left(Z_{2 n-1}, Z_{2 n}, k t\right), \delta\left(Z_{2 n}, Z_{2 n+1}, k t\right)\right) \\
& =\phi\left(V_{2 n-1}(k t), V_{2 n-1}(k t), V_{2 n}(k t)\right) .
\end{aligned}
$$

We prove that $V_{2 n}(k t) \geq V_{2 n-1}(k t)$. Now, if $V_{2 n}(k t)<V_{2 n-1}(k t)$ for some $n \in \mathbb{N}$, since $\phi$ is an increasing function, then the last inequality above we get

$$
V_{2 n}(t) \geq \phi\left(V_{2 n}(k t), V_{2 n}(k t), V_{2 n}(k t)\right)>V_{2 n}(k t) .
$$

That is $V_{2 n}(t)>V_{2 n}(k t)$, by Lemma1.12 this is a contradiction. Hence $V_{2 n}(k t) \geq$ $V_{2 n-1}(k t)$. Similarly, one can show that

$$
V_{2 n+1}(k t) \geq V_{2 n}(k t) .
$$

Then we deduce that

$$
V_{0}(t) \leq V_{1}(t) \leq V_{2}(t) \leq \cdots
$$

Thus $\left\{V_{n}(t)\right\}$ is increasing sequence in $[0,1]$. Therefore, tends to a limit $a \leq 1$. We claim that $a=1$. For if $a<1$ on making $n \rightarrow \infty$ the following inequality,

$$
\delta\left(Z_{n}, Z_{n+1}, t\right)=V_{2 n}(t) \geq \phi\left(V_{2 n-1}(k t), V_{2 n-1}(k t), V_{2 n-1}(k t)\right),
$$

we get

$$
a \geq \phi(a, a, a)>a,
$$

is a contradiction. Hence $a=1$, i.e.,

$$
V_{n}(t)=\delta\left(Z_{n}, Z_{n+1}, t\right) \longrightarrow 1 \text {. }
$$

It is easily seen that

$$
V_{n}(t) \geq V_{n-1}(k t) \geq \cdots \geq V_{0}\left(k^{n} t\right)=\delta\left(Z_{0}, Z_{1}, k^{n} t\right) .
$$


By the above inequality, then if $z_{n}$ is an arbitrary point in the set $Z_{n}$, for $n=0,1,2, \cdots$, it follows that

$$
M\left(z_{n}, z_{n+1}, t\right) \geq M\left(z_{0}^{\prime}, z_{1}^{\prime}, k^{n} t\right)
$$

for $z_{0}^{\prime} \in Z_{0}$ and $z_{1}^{\prime} \in Z_{1}$. Thus for some $a \in A$ and $b \in B$ if we set

$$
M(a, b, t)=\inf \{M(x, y, t) ; x, y \in X\} .
$$

Then $M\left(z_{n}, z_{n+1}, t\right) \geq M\left(a, b, k^{n} t\right)$.

By Lemma 1.8, the sequence $\left\{z_{n}\right\}$, and hence any sequence of $Z_{n}$, is a Cauchy sequence in $X$.

Suppose that $J(X)$ is complete. But $J x_{2 n+1} \in F x_{2 n}=Z_{2 n}$, for $n=$ $0,1,2, \cdots$. Therefore by the above, the sequence $\left\{J x_{2 n+1}\right\}$ is Cauchy and hence $J x_{2 n+1} \longrightarrow p=J v \in J(X)$, for some $v \in X$. But $I x_{2 n} \in G x_{2 n-1}=Z_{2 n-1}$ that is ,

$$
M\left(I x_{2 n}, J x_{2 n+1}, t\right) \geq \delta\left(Z_{2 n-1}, Z_{2 n}, t\right)=V_{2 n-1}(t) \longrightarrow 1 .
$$

Consequently, $I x_{2 n} \longrightarrow p$. Moreover, we have for $n=1,2,3, \cdots$

$$
\begin{aligned}
\delta\left(F x_{2 n}, p, t\right) & \geq \delta\left(F x_{2 n}, I x_{2 n}, \frac{t}{2}\right) * \delta\left(I x_{2 n}, p, \frac{t}{2}\right. \\
& \geq \delta\left(Z_{2 n}, Z_{2 n-1}, \frac{t}{2}\right) * \delta\left(I x_{2 n}, p, \frac{t}{2}\right) .
\end{aligned}
$$

Therefore, $\lim _{n \rightarrow \infty} \delta\left(F x_{2 n}, p, t\right)=1 * 1=1$. Hence from Lemma 1.14 it follows that $\lim _{n \rightarrow \infty} F x_{2 n}=\{p\}$. In like manner it follows that $\delta\left(G x_{2 n-1}, p, t\right) \longrightarrow 1$.

Since, for $n=1,2,3, \cdots$,

$$
\delta\left(F x_{2 n}, G v, t\right) \geq \phi\left(M\left(I x_{2 n}, J v, k t\right), \delta\left(I x_{2 n}, F x_{2 n}, k t\right), \delta(J v, G v, k t)\right) .
$$

On making $n \longrightarrow \infty$ if $G v \neq p$, then

$$
\begin{aligned}
\delta(p, G v, t) & >\phi(M(p, J v, k t), \delta(p, p, k t), \delta(J v, G v, k t)) \\
& >\delta(p, G v, k t) .
\end{aligned}
$$

Hence $\delta(p, G v, t)>\delta(p, G v, k t)$, by Lemma1.12 this is a contradiction. Thus $G v=\{p\}=\{J v\}$, since $G v \subseteq I X$, so $u \in X$ exists such that $\{I u\}=G v=$ $\{J v\}$. Now if $F u \neq G v$, then

$$
\begin{aligned}
\delta(F u, G v, t) & \geq \phi(M(I u, J v, k t), \delta(I u, F u, k t), \delta(J v, G v, k t)) \\
& >\delta(F u, G v, k t),
\end{aligned}
$$

by Lemma1.12 this is a contradiction. So we have $F u=G v$. Hence $F u=$ $\{p\}=G v=\{I u\}=\{J v\}$. Since $F u=\{I u\}$ and the pair $\{F, I\}$ is weakly compatible, we obtain $F p=F I u=I F u=\{I p\}$. If $F p \neq\{p\}$, we have

$$
\begin{aligned}
\delta(F p, p, t) & =\delta(F p, G v, t) \\
& \geq \phi(M(I p, J v, k t), \delta(I p, F p, k t), \delta(J v, G v, k t)) \\
& >\delta(F p, p, k t),
\end{aligned}
$$


by Lemma1.12 this is a contradiction. It follows that $F p=\{I p\}=\{p\}$. Similarly, $G p=\{J p\}=\{p\}$. Therefore, we obtain $F p=G p=\{J p\}=\{I p\}=$ $\{p\}$. To see the $p$ is unique, suppose that $\{q\}=\{I q\}=\{J q\}=F q=G q$. If $p \neq q$, then

$$
\begin{aligned}
M(p, q, t) & \geq \delta(F p, G q, t) \\
& \geq \phi(M(I p, J q, k t), \delta(I p, F p, k t), \delta(J q, G q, k t)) \\
& >M(p, q, k t),
\end{aligned}
$$

is a contradiction. It follows that $p=q$.

\section{REFERENCES}

[1] El Naschie MS. On the uncertainty of Cantorian geometry and two-slit experiment. Chaos, Solitons and Fractals 1998; 9:517-29.

[2] El Naschie MS. A review of $E$-infinity theory and the mass spectrum of high energy particle physics. Chaos, Solitons and Fractals 2004; 19:209-36.

[3] El Naschie MS. On a fuzzy Kahler-like Manifold which is consistent with two-slit experiment. Int J of Nonlinear Science and Numerical Simulation 2005; 6:95-8.

[4] El Naschie MS. The idealized quantum two-slit gedanken experiment revisitedCriticism and reinterpretation. Chaos, Solitons and Fractals 2006; 27:9-13.

[5] Fisher B, Common fixed points of mappings and set-valued mappings, Rostock. Math. Kolloq. 18(1981), 69-77.

[6] Fisher B, Sessa S. Two common fixed point theorems for weakly commuting mappings, Period. Math. Hungar. 20(1989), 207-218.

[7] George A, Veeramani P. On some result in fuzzy metric space. Fuzzy Sets Syst 1994; 64:395-9.

[8] Gregori V, Sapena A. On fixed-point theorem in fuzzy metric spaces. Fuzzy Sets and Sys 2002; $125: 245-52$.

[9] Jungck G and Rhoades B. E, Some fixed point theorems for compatible maps, Internat. J. Math. Math. Sci.16(1993), 417-428.

[10] Jungck G and Rhoades B. E, Fixed points for set valued functions without continuity, Indian J. Pure Appl. Math. 29(1998), no. 3,227-238.

[11] Kramosil I, Michalek J. Fuzzy metric and statistical metric spaces. Kybernetica 1975; 11:326-34.

[12] Rodríguez López J, Ramaguera S. The Hausdorff fuzzy metric on compact sets. Fuzzy Sets Sys 2004; 147:273-83.

[13] Mihet D. A Banach contraction theorem in fuzzy metric spaces. Fuzzy Sets Sys 2004; 144:431-9.

[14] Schweizer B, Sherwood H, and Tardiff RM. Contractions on PM-space examples and counterexamples. Stochastica 1988; 1:5-17.

[15] Turkoglu D , Altun I and Cho Y. J. Common Fixed points of Compatible Mappings of Type I and II in Fuzzy Metric Spaces, J.Fuzzy Math., accepted "

[16] Tanaka Y, Mizno Y, Kado T. Chaotic dynamics in Friedmann equation. Chaos, Solitons and Fractals 2005; 24:407-22.

[17] Zadeh LA. Fuzzy sets. Inform and Control 1965; 8:338-53.

\section{Received: October 14, 2006}

